\title{
Triaxiality of the ground states in the ${ }^{174} \mathbf{W}$
}

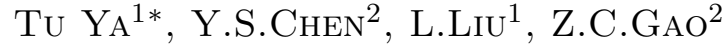

${ }^{1}$ College of Physics science and Technology, ShenYang Normal University, ShenYang 110034, P.R. China

${ }^{2}$ China Institute of Atomic Energy, Beijing 102413, P.R. China

\begin{abstract}
We have performed calculations for the ground states in ${ }^{174} \mathrm{~W}$ by using the projected total energy surface (PTES) calculations. Both the ground state (g.s.) band and its $\gamma$ band reproduce the experimental data. Further discussion about the triaxiality in ${ }^{174} \mathrm{~W}$ has been made by transition quardrupole moment $\left(Q_{t}\right)$ and comparing between the PTES and TRS methods.
\end{abstract}

\section{Introduction}

The triaxiality of highly rotating nuclei has been a substantial subject of study for several decades. Nuclear triaxiality associated with the breaking of axial symmetry of the quadrupole deformation, brings up many interesting collective motions, e.g., wobbling motion, chiral and $\gamma$ bands. And the role of the beyond-mean-field-effect on the nuclear triaxiality has become another hot topic.

several theoretical approaches have been developed for studying nuclear triaxiality $[1,2]$. However, much less research focus had been paid to the axial asymmetry of the nuclear ground state. Recently, the first global calculation across the nuclear chart of axial symmetry breaking was carried out by using the macroscopic-microscopic finite-range liquid-drop model (FRLDM) $[3,4]$.

*tuya_sy@126.com 
Many nuclear ground states have been predicted to be triaxiality shaped or $\gamma$-soft by the FRLDM calculations and, however, some of which were previously predicted to be axial symmetric by the total routhian surface (TRS) method. TRS method has been extensively used to describe the shape of highly rotating nuclei. Despite the many success of the TRS in the description of nuclear shapes, the disadvantages are clear in the fact that the angular momentum is not a good quantum number, but instead the rotational frequency is employed. And the assumption of the fixed rotational axis in the TRS method is unreasonable in the description of the triaxial nuclear system. The different results of the FRLDM from the TRS method come from their different parameterizations although the two methods are belong to the same type of macroscopic-microscopic approach.

Recently, the PTES method has been developed based on the triaxial projected shell model (TPSM) hybridized with the macroscopic-microscopic method [5], which describes the total energy surface of a triaxially deformed nucleus with the good angular momentum quantum number. To overcome the shortcomings of the TRS method the classical rotational term is replaced by the full quantum mechanics rotational energy in the PTES. The total energy of an atomic nucleus is decomposed into the macroscopic, microscopic and rotational terms. The macroscopic and microscopic parts are described with the liquid drop model and Strutinsky method, respectively, and the rotational energy is given by the TPSM, as the beyond mean field term. In the present work, we will discussed the triaxiality in ${ }^{174} \mathrm{~W}$ by performing PTES method.

\section{Projected Total Energy Surface method}

In the PTES method, the total energy of the nuclear system includes three terms and is expressed as (see Ref [5] for details)

$$
E^{t o t}=E_{L D}+E_{c o r r}+E_{\text {rot }} .
$$

Where $E_{L D}$ is the liquid-drop model energy [6]. $E_{\text {corr }}$ is the quantum effect correction to the energy, which is given by the Strutinsky method $[7,8]$. $E_{r o t}$ is the rotational energy obtained by the TPSM calculation. All the terms in Eq.(1), of course, depend on the neutron and proton numbers $(N, Z)$, the deformation parameters, $\varepsilon_{2}, \gamma$ and $\varepsilon_{4}$, which are not written explicitly.

The TPSM wave function can be written as

$$
\left|\Psi_{I M}\right\rangle=\sum_{K \kappa} F_{\kappa, K}^{I} \hat{P}_{M K}^{I}\left|\Phi_{\kappa}\right\rangle,
$$


in which the projected multi-q.p. states span the shell model space. Where, $\left|\Phi_{\kappa}\right\rangle$ represents the set of multi-quasiparticle states labeled by $\kappa$, and for even-even nuclei it includes the 2- and 4-q.p, states associated with the triaxially deformed q.p. vacuum $|0\rangle, \quad \alpha_{\nu_{1}}^{\dagger} \alpha_{\nu_{2}}^{\dagger}|0\rangle, \quad \alpha_{\pi_{1}}^{\dagger} \alpha_{\pi_{2}}^{\dagger}|0\rangle, \quad \alpha_{\nu_{1}}^{\dagger} \alpha_{\nu_{2}}^{\dagger} \alpha_{\pi_{1}}^{\dagger} \alpha_{\pi_{2}}^{\dagger}|0\rangle$.

The rotational energies together with the wave functions, the coefficients $F_{\kappa, K}^{I}$, are obtained by solving the eigenvalue equation,

$$
\sum_{K \kappa} F_{\kappa K}^{I}\left(\left\langle\Phi_{\kappa^{\prime}}\left|H P_{K^{\prime} K}^{I}\right| \Phi_{\kappa}\right\rangle-E^{I}\left\langle\Phi_{\kappa^{\prime}}\left|P_{K^{\prime} K}^{I}\right| \Phi_{\kappa}\right\rangle\right)=0 .
$$

The Hamiltonian in the TPSM [see [] for details] is written as

$$
H=H_{0}-\frac{1}{2} \sum_{\lambda=2}^{4} \chi_{\lambda} \sum_{\mu=-\lambda}^{\lambda} Q_{\lambda \mu}^{\dagger} Q_{\lambda \mu}-G_{0} P_{00}^{\dagger} P_{00}-G_{2} \sum_{\mu=-2}^{2} P_{2 \mu}^{\dagger} P_{2 \mu} .
$$

The nuclear equilibrium deformation can be obtained for each spin by minimizing the total energy, calculated by Eq.(1), in function of spin with respect to the deformation parameters, $\varepsilon_{2}$ and $\gamma$. The minimization procedure can be performed through the calculation of the total energy surface in the considered deformation plan.

\section{Results of calculations and discussion}

Three major shells of $\mathrm{N}=4,5,6$ for neutrons and $\mathrm{N}=3,4,5$ for protons are included to calculate the Nilsson single particle states in the TPSM. The used Nilsson potential parameters for each main shells are taken from the literature [9]. In the present calculation, the hexadecapole deformation is not considered as the variable but taken to be $\varepsilon_{4}=0$ for simplicity. The monopole pairing strength parameter $G_{0}$ is calculated with the standard parameters $g_{1}=20.12 \mathrm{MeV}$ and $g_{2}=13.13 \mathrm{MeV}$ [10], which approximately reproduces the observed odd-even mass differences in the mass region. The quadrupole pairing strength parameter $G_{2}$ is calculated from $G_{2}=f G_{0}$ with the proportional coefficient $f=0.16$ as usual.

The projected total energy surface is calculated for an given angular momentum as well as a given parity, this may provides an opportunity to make a direct comparison between the theory and the experiment. Figure 1 shows the PTES for the g.s. $I^{\pi}=0^{+}$of ${ }^{174} \mathrm{~W}$, which has a local minimum representing the equilibrium deformations of $\left(\varepsilon_{2}=0.24, \gamma=18^{\circ}\right)$. The results imply that the g.s. of the nucleus is well deformed, and it is particularly 


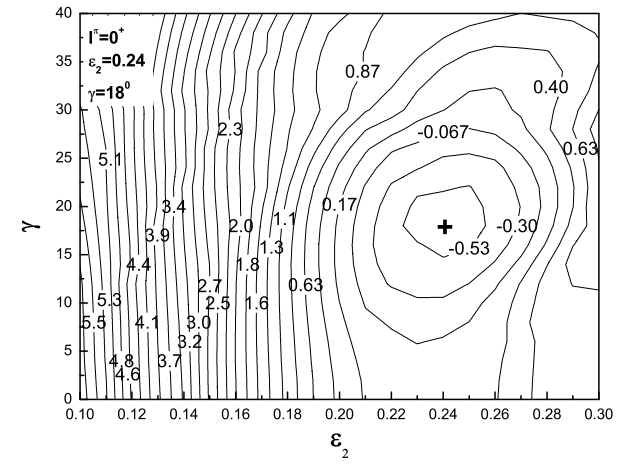

Figure 1: The contour plot of total energy in units of $\mathrm{MeV}$ for the ground state in ${ }^{174} \mathrm{~W}$, the minimum is marked by "+".

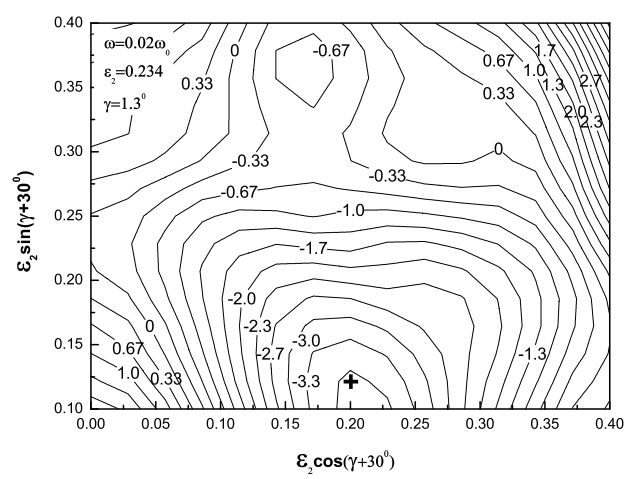

Figure 2: The contour plot of total routhian in units of $\mathrm{MeV}$ for ${ }^{174} \mathrm{~W}$, calculated at the rotational frequency $\hbar \omega=0.02 \hbar \omega_{0}$, the minimum is marked by "+".

noteworthy to have the axial asymmetry with $\gamma=18^{\circ}$. The TRS calculation has been intensively employed to study highly rotating nuclei [1],especially, in the rare earth mass region, thus it may be instructive to make a comparison between the PTES and TRS results. Because of the semi-classical nature of the TRS method it is impossible to strictly describe the g.s. which has a good angular momentum $I^{\pi}=0^{+}$. However, the TRS calculated at a small rotational frequency may describe the property of the g.s. as a good approximation, and thus a comparison between the PTES and TRS for the g.s. has the certain significance. Figure 2 shows the TRS calculated with the same set of the Nilsson potential parameters for the ground band state of ${ }^{174} \mathrm{~W}$, at the rotational frequency of $\hbar \omega=0.02 \hbar \omega_{0}$, which reports a local minimum at the deformations of $\left(\varepsilon_{2}=0.234, \gamma \sim 0^{\circ}\right)$, which consistent with the result of FRLDM. The three methods give the same equilibrium elongation deformation but a considerably different triaxiality for the g.s. of ${ }^{174} \mathrm{~W}$. The $\gamma$-deformation of $18^{\circ}$ is not a large triaxiality but an indication of the axial symmetry breaking in the g.s. of the nucleus. In fact, the axial asymmetry of the g.s. of ${ }^{174} \mathrm{~W}$ manifests the appearance of the characteristic $\gamma$ band observed experimentally at $0.977 \mathrm{MeV}$ above the g.s.. The calculations of the PTES for nonzero spins of g.s. band and its $\gamma$ band of ${ }^{174} \mathrm{~W}$, have been performed in a similar way to the g.s. calculation. Figure 3 presents the calculated g.s. band and its $\gamma$ band for ${ }^{174} W$, from the PTES minima, compared with the experimental data [11]. By comparison, it is seen that the beyond mean field effects incorporated in the PTES approach may give rise to the axial asymmetry in some nuclei which have been predicted to 


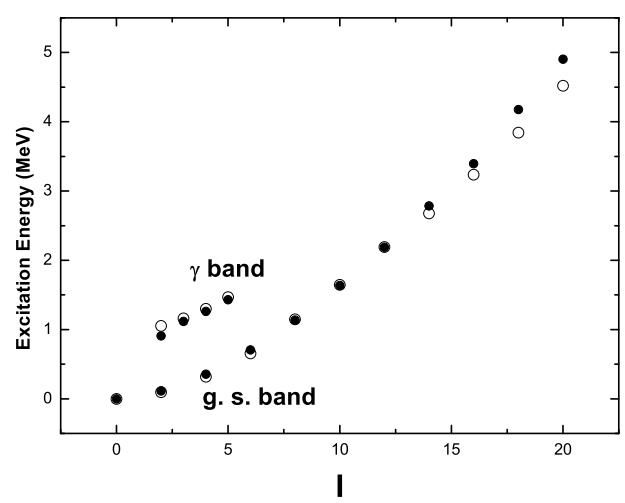

Figure 3: Calculated g.s. band and its $\gamma$ band (open circle), the energy versus spin, for ${ }^{174} \mathrm{~W}$, from the PTES minima, compared with the experimental data [11] (solid circle).

have an axial symmetric shape by the TRS calculations and FRLDM calculation. In another way, the transition quadrupole moment $\left(Q_{t}\right)$ is one of best physical quantities to identify the intrinsic deformed shape of the nucleus. The calculated $Q_{t}$ with the deformations determined by minimizing the projected total energies for different spin, well reproduce the available experimental data $[12,13]$ within the error bars for $W$ isotopes (see [5] and next work). Unfortunately, there are no more clear experimental data for ${ }^{174} \mathrm{~W}$ to compare with the calculations.

\section{Summary}

In summary, we have studied the triaxiality of the ground states in ${ }^{174} \mathrm{~W}$ by using PTES method. Our PTES calculations and the comparison with TRS method shows that beyond-mean-field-effect may play an important role on the nuclear triaxial deformation.

\section{Acknowledgements}

The work is supported by National Nature Science Foundation of China under Grant Nos.11047171, 11301508, 11175258, 11021504, 11275067,11575290 , and by the Knowledge Innovation Project of the Chinese Academy of Sciences under Grant No.KJCX2-SW-N02, the key project of Science and Technology Research of Education Ministry of China under grant number 209053. 


\section{References}

[1] Tu Ya and Y.S.Chen, et al., Nucl. Phys. A848, 260(2010).

[2] Z.C. Gao, Y.S.Chen, Y.Sun, Phys. Lett. B634, 195(2006).

[3] P. Möller and R. Bengtsson et al., Phys. Rev. Lett.97, 162502(2006).

[4] P.Möller, A.J. Sierk, R. Bengtsson, H.Sagawa and T. Ichikawa, Atomic Data and Nuclear Data Tables 98, 149(2012).

[5] Tu Ya and Y.S.Chen et al.,Sci. China Ser G-Phys. Mech. Astron. 57(11), 2054(2014).

[6] W.D.Myers and W. Swiatecki, Ark. Fys.361,343(1967).

[7] V.M.Strutinsky, Nucl. Phys. A122 , 1(1968).

[8] V.M.Strutinsky, Nucl. Phys. A85, 420(1967).

[9] T. Bengtsson and I.Ragnarsson, Nucl. Phys. A436, 14(1985).

[10] K.Hara and Y.Sun, Int. J. Mod. Phys. E4, 637(1995).

[11] T.Kibédi et al., Nucl. Phys. A688, 669(2001).

[12] F.K.Mcgowan and N.R.Johnson et al., Nucl. Phys. A530, 490(1991).

[13] F.K.Mcgowan and N.R.Johnson et al., Nucl. Phys. A580, 335(1994). 